\title{
Responsabilidad universitaria en la implementación de los objetivos de desarrollo sostenible
}

\author{
Sara Serrate González, Judith Martín Lucas, David Caballero Franco, \\ y José Manuel Muñoz Rodríguez \\ Universidad de Salamanca (España)
}

Las instituciones de educación superior deben favorecer la implementación de la Agenda 2030 para el Desarrollo Sostenible aprobada en las Naciones Unidas, y una forma de promoverlo es proporcionar a los estudiantes una conciencia de Sostenibilidad basada en competencias para que puedan resolver los numerosos desafíos que encontrarán en sus campos profesionales. En este trabajo se presentan los principales resultados del primero y cuarto objetivo propuesto en el Proyecto EDINSOT, cuyo fin principal es la formación de egresados en competencias en Sostenibilidad en el Sistema Universitario Español. Se proporciona una revisión de las competencias genéricas en Sostenibilidad en los títulos de educación de nueve universidades españolas y un mapa de competencias que permite evaluar los estudios universitarios y sus títulos, así como un análisis de la percepción del alumnado respecto a su nivel de formación adquirido a lo largo de la carrera en cuestiones de sostenibilidad en los Grados de Pedagogía y Educación Social de la Universidad de Salamanca. Nos centraremos en las aportaciones más relevantes sobre su visión y valoración de la sostenibilidad, su experiencia formativa en la universidad, así como sus propuestas sobre cómo podría mejorarse, tanto desde el punto de vista metodológico como de organización curricular.

Palabras clave: EDINSOST, sostenibilidad, educación, universidad.

University responsability in the implementation of the Sustainable Development Goals. The adoption of the 2030 Agenda for Sustainable Development approved by United Nations should be promoted by higher education institutions. A great way to achieve this goal is to foster awareness on sustainability and development to undergraduate students considering their different studies areas. This paper present results obtained from the first and forth objectives from EDINSOST project. EDINSOST is a Spanish project focused on training in Sustainability in higher educational Spanish system. In this case, the results provided come from the general competences on Sustainability from nine different Spanish universities. In this case we focus on the review of the general competences on Sustainability and a competences map which allows us to assess the different degree studies. Additionally, we will focus on the results from the perception of student's analysis regarding Sustainability in Pedagogy and Social Education degrees. Focusing on the most relevant contributions related view and assessment of Sustainability, their formative experience within university and their improvement proposals regarding methodology and curricular organization.

Keywords: EDINSOST, sustainability, education, university.

Correspondencia Sara Serrate González. Avenida Francisco Tomás y Valiente s/n. C.P.: 37007 Salamanca (España). E-mail: $\underline{\text { sarasg@ @usal.es. }}$ 
Nos encontramos ante un mundo caracterizado por procesos globales, guiados en primera instancia por los procesos económicos, un contexto complejo del que forman parte las desigualdades, las guerras, la desertificación, el cambio climático o las crisis económicas. No obstante, las Naciones Unidas (2014) ya afirmaban que tenemos los conocimientos y medios necesarios para hacer frente a algunos de los retos a los que se enfrenta la sociedad del siglo XXI. Por otro lado, y como afirman Martín y Caballero (2017) las instituciones públicas serán las encargadas de liderar este nuevo camino. Las Universidades como instituciones públicas y educativas no pueden quedar al margen de los retos que plantean estos nuevos contextos y deben tomar las riendas en la implementación de la Agenda 2030. La Universidad debe ir más allá de educar profesionales, tiene el deber de formar a ciudadanos con conciencia ética y compromiso cívico vinculando la sostenibilidad a esta formación (Michelsen, 2016). Este tipo de formación y de apertura a la comunidad permite que la sociedad perciba la Universidad como una institución cercana, con capacidad de transformar la sociedad misma realizando de forma práctica valores que nos hacen a todos más humanos (Núñez, Calvo, y Álvarez, 2015).

Ante estos retos, es necesario incorporar la responsabilidad y el compromiso que implica la ciudadanía global en la vida cotidiana (CONGCYL, 2014). Aunque la definición del término Desarrollo Sostenible sigue generando cierta controversia, existe un consenso mundial sobre la necesidad de una educación que permita crear conciencias críticas y ciudadanos activos que puedan hacer frente a los retos que nos plantea la sociedad actual (Naciones Unidas, 2012). Por tanto, la educación para el desarrollo se hace necesaria en una sociedad global como la que vivimos (Caride, 2017), pues, desde su disposición transformadora persigue como principal objetivo promover una ciudadanía generadora de soluciones globales ante los retos que nos plantea el siglo XXI, un tipo de educación que debe promover y potenciar el desarrollo de una conciencia de ciudadanía global, que haga a los sujetos protagonistas responsables, solidarios, críticos y activos en un desarrollo humano sostenible en el que todos estamos implicados, generando una conciencia planetaria comprometida en la lucha contra las grandes desigualdades (Aznar y Barrón, 2017; Boni, Hofmann, y Sow, 2012; Ortega, 2007). En definitiva, una educación promotora del Desarrollo Humano y Sostenible (Sainz, 2016). Volviendo al tema que nos preocupa, es indiscutible que la educación, y en concreto, la educación superior se constituye como mecanismo clave hacia la sensibilización y formación para los cambios y transformaciones demandados por la Agenda 2030, no obstante, ¿están las universidades preparadas para afrontar estos retos? ¿Conoce el profesorado y por lo tanto el alumnado las competencias en sostenibilidad en relación con la formación universitaria? ¿Se está preparando en clave de sostenibilidad a los profesionales del presente y futuro? 
Intentando dar respuesta a estas cuestiones, Segalas y Sánchez (2019) muestran cómo los alumnos consideran que no se trabaja de manera adecuada las competencias en sostenibilidad. Lo achacan en mayor medida a la falta de concienciación y por tanto motivación del profesorado. Este último debe ser un estamento clave en la universidad para que se produzca un cambio integral en la estructura universitaria en cuando al desarrollo sostenible. Los alumnos que están liderando este cambio en la sociedad actual piensan en que la formación del profesorado es el principal problema para que se trabaje los objetivos de desarrollo sostenible desde la transversalidad (Valderrama-Hernández et al., 2019). El alumnado no percibe que sus profesores estén preparados para entender la sostenibilidad, por lo tanto, mucho menos para integrarla en las clases de manera transversal o de manera específica. La visión de las alumnas y alumnos en diversas Universidades de España es común en cuanto al profesorado.

Con lo cual, si queremos ofrecer una formación de calidad desde el ámbito de la educación superior, y atendiendo a las directrices y objetivos recogidos en la Agenda 2030 se hace necesario dar respuesta por parte del profesorado, principalmente a estas y otras cuestiones. Así es como nace el proyecto EDINSOST, destinado a fomentar un cambio hacia una sociedad más sostenible desde la institución universitaria que presentamos a continuación.

\section{El proyecto EDINSOST}

El proyecto EDINSOST "Educación e Innovación Social para la Sostenibilidad. Formación en las Universidades españolas de profesionales como agentes de cambio para afrontar los retos de la sociedad" parte de la base del contexto social, político, económico y medioambiental actual, que demanda una ciudadanía crítica y responsable una conciencia planetaria y sea capaz de asumir la responsabilidad y compromiso de cambio hacia un mundo más sostenible. El proyecto pretende buscar respuestas a los retos que plantea actualmente la sociedad en pro de poder orientar políticas de promoción y de investigación que permitan abordarse a través del sistema educativo. Además de formar a los diferentes profesionales desde las Universidades españolas como agentes de cambio para afrontar los retos de la sociedad (Sánchez et al., 2017). El objetivo principal de este proyecto es avanzar en la innovación educativa en la Educación para el Desarrollo en las Universidades Españolas con el fin de dotar a los futuros titulados de competencias que permitan generar un cambio hacia una sociedad más sostenible. Para poder lograr este objetivo se cuenta con la colaboración y trabajo interdisciplinar de diferentes universidades españolas de las cuales participan un total de quince titulaciones de los ámbitos de ingeniería y educación.

Para impulsar el cambio hacia la sostenibilidad desde la Sostenibilización Curricular es necesario tener en cuenta algunos aspectos como la importancia de 
introducir competencias de carácter transversal relacionadas con la sostenibilidad dentro del currículum de las diferentes carreras universitarias, así como trabajar desde metodologías características de la Educación para el Desarrollo. Por todo ello, el proyecto EDINSOST se divide en cuatro objetivos específicos:

-Definir el mapa de sostenibilidad de las titulaciones participantes y establecer el marco que facilite su integración en los estudios de manera holística.

-Validar estrategias didácticas para la adquisición de la sostenibilidad desde un enfoque pedagógico constructivista y comunitario.

-Diagnosticar el estado de las necesidades formativas en sostenibilidad en el profesorado y elaborar y ensayar propuestas de capacitación.

-Diagnosticar el estado de aprendizaje de la sostenibilidad en el alumnado universitario y elaborar y ensayar propuestas de capacitación.

Estos objetivos nos permitirán visualizar cómo se materializa la educación para el desarrollo sostenible en el currículum universitario a través del análisis de las prácticas pedagógicas y el desarrollo de las competencias en la formación universitaria.

\section{MÉTODO}

\section{Participantes}

La muestra participante se compuso de un total de 115 estudiantes de la Facultad de Educación de la Universidad de Salamanca, con edades comprendidas entre los 18 y los 30, con una edad media de 21,07 años. Por cursos, el 66,1\% eran estudiantes de primero y el $33,9 \%$ estudiantes de cuarto. En cuanto al sexo, un $95 \%$ eran mujeres.

\section{Instrumentos}

Para abordar el objetivo 1, se utilizó un mapa de competencias representado en la tabla 1, instrumento creado y validado dentro de este proyecto con el objetivo evaluar e integrar la sostenibilidad en el sistema universitario (Andrades, Larragán, y Muriel, 2018; Larrán, Herrera, y Andrades, 2015; Murga-Menoyo y Novo, 2015; Segalas y Sánchez, 2019).

El mapa nos permite analizar las cuatro competencias relacionadas con los objetivos del proyecto y en función a diferentes niveles de dominio (saber, saber cómo y demostrar + hacer):

-C1: Contextualización crítica del conocimiento estableciendo interrelaciones con la problemática social, económica y ambiental, local y/o global;

-C2: Utilización sostenible de recursos y prevención de impactos negativos sobre el medio natural y social;

-C3: Participación en procesos comunitarios que promuevan la sostenibilidad; 
-C4: Aplicación de principios éticos relacionados con los valores de la sostenibilidad en los comportamientos personales y profesionales.

Tabla 1. Mapa de competencias en sostenibilidad

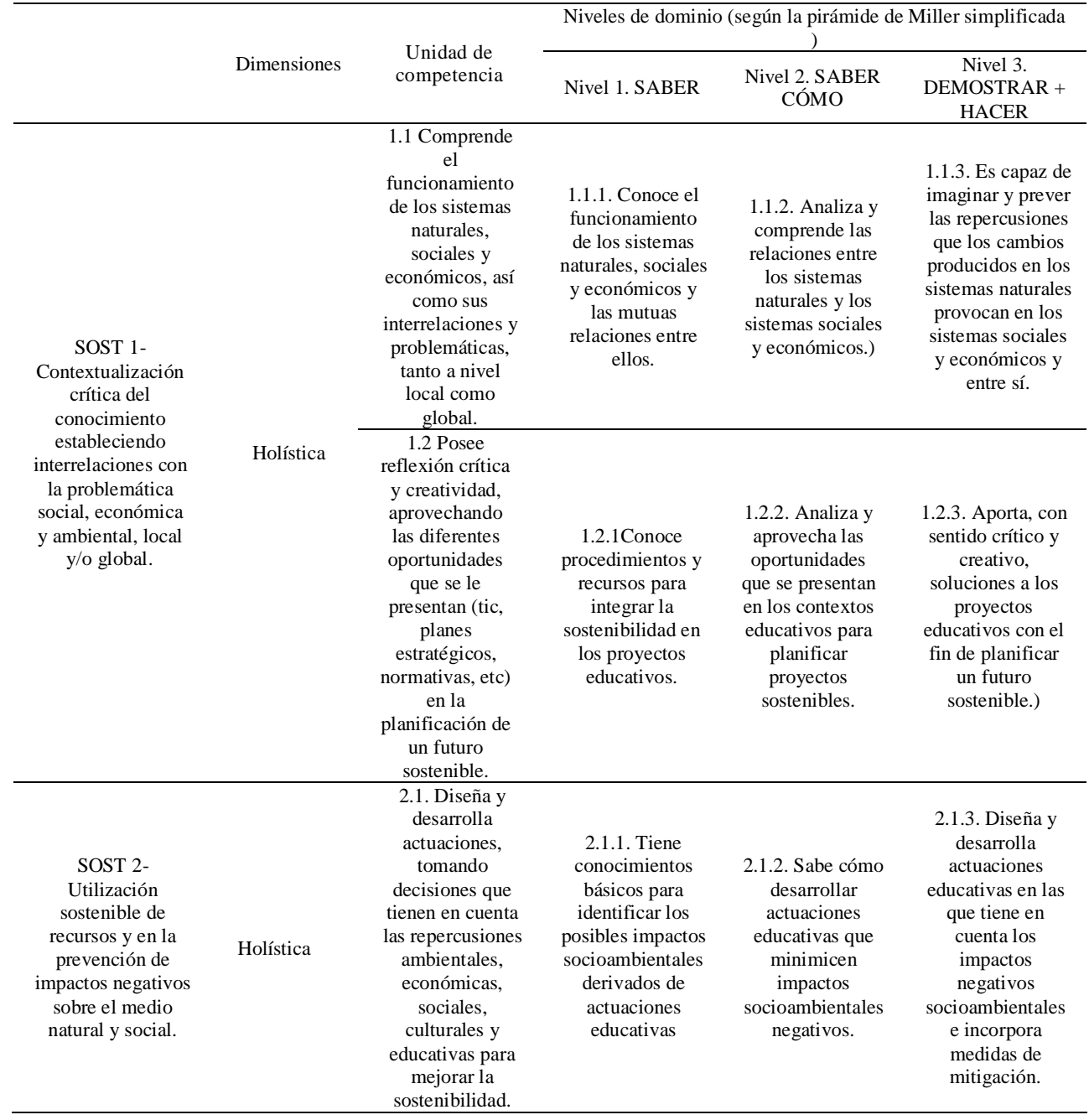


SERRATE et al. Responsabilidad universitaria en los ODS

Tabla 1 (continuación). Mapa de competencias en sostenibilidad

\begin{tabular}{|c|c|c|c|c|c|}
\hline & \multirow[b]{2}{*}{ Dimensiones } & \multirow[b]{2}{*}{$\begin{array}{l}\text { Unidad de } \\
\text { competencia }\end{array}$} & \multicolumn{3}{|c|}{ Niveles de dominio (según la pirámide de Miller simplificada ) } \\
\hline & & & Nivel 1. SABER & $\begin{array}{l}\text { Nivel 2. SABER } \\
\text { CÓMO }\end{array}$ & $\begin{array}{c}\text { Nivel } 3 . \\
\text { DEMOSTRAR + } \\
\text { HACER } \\
\end{array}$ \\
\hline $\begin{array}{c}\text { SOST 3- } \\
\text { Participación en } \\
\text { procesos } \\
\text { comunitarios que } \\
\text { promuevan la } \\
\text { sostenibilidad. }\end{array}$ & Holística & $\begin{array}{l}\text { 3.1 Promueve y } \\
\text { participa en } \\
\text { procesos } \\
\text { comunitarios que } \\
\text { fomentan la } \\
\text { sostenibilidad. }\end{array}$ & $\begin{array}{c}\text { 3.1.1. Se } \\
\text { reconoce como } \\
\text { parte integrante } \\
\text { de su entorno y } \\
\text { conoce } \\
\text { programas } \\
\text { educativos } \\
\text { comunitarios } \\
\text { que fomentan la } \\
\text { participación y } \\
\text { el compromiso } \\
\text { en la mejora } \\
\text { socioambiental. }\end{array}$ & $\begin{array}{l}\text { 3.1.2. Sabe } \\
\text { desenvolverse } \\
\text { de manera } \\
\text { satisfactoria en } \\
\text { proyectos } \\
\text { educativos } \\
\text { comunitarios, } \\
\text { fomentando la } \\
\text { participación. }\end{array}$ & $\begin{array}{l}\text { 3.1.3. Diseña y lleva } \\
\text { a cabo actividades } \\
\text { socioeducativas en } \\
\text { procesos } \\
\text { comunitarios } \\
\text { participativos que } \\
\text { promueven la } \\
\text { sostenibilidad. }\end{array}$ \\
\hline \multirow{2}{*}{$\begin{array}{c}\text { SOST 4- } \\
\text { Aplicación de } \\
\text { principios éticos } \\
\text { relacionados con } \\
\text { los valores de la } \\
\text { sostenibilidad en } \\
\text { los } \\
\text { comportamientos } \\
\text { personales y } \\
\text { profesionales }\end{array}$} & \multirow[t]{2}{*}{ Holística } & $\begin{array}{l}\text { 4.1. Es coherente en } \\
\text { sus actuaciones, } \\
\text { respetando y } \\
\text { valorando la } \\
\text { diversidad } \\
\text { (biológica, social y } \\
\text { cultural) y } \\
\text { comprometiéndose } \\
\text { con la mejora de la } \\
\text { sostenibilidad. }\end{array}$ & $\begin{array}{l}\text { 4.1.1. Conoce los } \\
\text { principios éticos } \\
\text { de la } \\
\text { sostenibilidad y } \\
\text { la importancia del } \\
\text { respeto a la } \\
\text { diversidad en la } \\
\text { intervención } \\
\text { educativa. }\end{array}$ & $\begin{array}{c}4.1 .2 . \\
\text { Comprende e } \\
\text { integra los } \\
\text { principios } \\
\text { éticos de } \\
\text { sostenibilidad } \\
\text { en sus acciones, } \\
\text { considerando la } \\
\text { naturaleza un } \\
\text { bien en sí } \\
\text { mismo y } \\
\text { transmitiendo la } \\
\text { importancia de } \\
\text { la educación } \\
\text { para un cambio } \\
\text { en la relación } \\
\text { del ser humano } \\
\text { con el medio } \\
\text { social y } \\
\text { cultural. }\end{array}$ & $\begin{array}{l}\text { 4.1.3. Es capaz de } \\
\text { diseñar y/o gestionar } \\
\text { proyectos educativos } \\
\text { tomando en } \\
\text { consideración la } \\
\text { ética ecológica, para } \\
\text { mejorar la calidad de } \\
\text { vida y promover el } \\
\text { bien común. }\end{array}$ \\
\hline & & $\begin{array}{l}\text { 4.2. Promueve una } \\
\text { educación en } \\
\text { valores orientada a } \\
\text { la formación de una } \\
\text { ciudadanía } \\
\text { responsable, activa } \\
\text { y democrática. }\end{array}$ & $\begin{array}{l}\text { 4.2.1. Considera } \\
\text { la promoción del } \\
\text { desarrollo } \\
\text { humano, integral } \\
\text { y sostenible, } \\
\text { como una } \\
\text { finalidad } \\
\text { fundamental de } \\
\text { la formación } \\
\text { ciudadana. }\end{array}$ & $\begin{array}{c}\text { 4.2.2. Analiza } \\
\text { críticamente y } \\
\text { valora las } \\
\text { consecuencias } \\
\text { que su actuación } \\
\text { personal y } \\
\text { profesional } \\
\text { puede tener en } \\
\text { el desarrollo } \\
\text { integral de los } \\
\text { educandos y en } \\
\text { la promoción de } \\
\text { un desarrollo } \\
\text { humano } \\
\text { sostenible. }\end{array}$ & $\begin{array}{l}\text { 4.2.3. Diseña y } \\
\text { desarrolla } \\
\text { propuestas de } \\
\text { intervención } \\
\text { educativa que } \\
\text { integren valores de } \\
\text { sostenibilidad y } \\
\text { redunden en la } \\
\text { justicia y el bien } \\
\text { común. }\end{array}$ \\
\hline
\end{tabular}

En cuanto al objetivo 4 el instrumento utilizado fue un cuestionario destinado a estudiantes de Grado, compuesto por 18 ítems (escala Likert 1 Totalmente en Desacuerdo-4 Totalmente de Acuerdo) con los que se ofrece una definición amplia de sostenibilidad incluyendo sus tres dimensiones: social, medioambiental y económica. 


\section{Procedimiento}

Para abordar el objetivo 1, se procedió a realizar un análisis pormenorizado de las distintas materias que componen los Grados de Pedagogía y Educación Social, concretamente de la Universidad de Salamanca, a través de las memorias académicas y los documentos de verificación de las titulaciones. Específicamente se realizó un análisis a través de las guías docentes buscando comprobar si se trabajan aspectos relacionados con la sostenibilidad en las competencias propias de la titulación de Pedagogía y Educación Social, en los objetivos, temario, actividades, y proceso de evaluación de las distintas materias que componen los Grados. Una vez obtenidos los datos pertinentes, se analizaron en base a los criterios del mapa de competencias presentado en la tabla 1.

Respecto al objetivo 4, se optó por utilizar una metodología cuantitativa, con un diseño de investigación de tipo pre-experimental con pre-test y pos-test (Campbell y Standley, 1963). Se utilizó un método descriptivo-inferencial, por medio del estudio de encuesta electrónica aplicada a los estudiantes de primero antes de comenzar las materias del primer cuatrimestre y a los estudiantes de cuarto una vez finalizada la formación universitaria.

El tratamiento de los datos se centró en un estudio descriptivo, por medio del análisis de medias sobre las percepciones que tenían los estudiantes antes de comenzar su formación universitaria respecto a los conocimientos en sostenibilidad. También se realizó un análisis de tipo inferencial a partir de una comparación de medias utilizando la prueba paramétrica de contraste de hipótesis t de Student para muestras independientes y para muestras relacionadas, además de la Prueba ANOVA; ambas permitieron comprobar la existencia de diferencias estadísticamente significativas en cuanto a las valoraciones recogidas en las dos aplicaciones (alumnos de primero y alumnos de cuarto).

Del mismo modo se comprobó la existencia de diferencias en función la titulación y en función de si los estudiantes habían participado con anterioridad en proyectos de sostenibilidad o habían recibido formación docente previa.

\section{RESULTADOS}

\section{El mapa de competencias}

El estudio pormenorizado basado en el mapa de competencias nos permite afirmar que la formación recibida permite al estudiante situarse en el nivel de dominio "Saber", siendo pocos los ejercicios que permiten trascender al "Saber Cómo" que sería el nivel 2 de dominio o al "Demostrar y Hacer" que suponen el nivel 3. Tan solo determinadas materias vinculadas directamente a los estudios en sostenibilidad como Pedagogía Ambiental, del Grado en Pedagogía o Educación Socio ambiental del Grado en Educación Social facilitan el nivel 2 y 3 de dominio. 
Por su parte, este nivel de dominio adquirido es resultado de la puesta en práctica de actividades y tareas vinculadas a una conexión directa con la sociedad, como las prácticas de aprendizaje-servicio o trabajo por proyectos. En las materias en las que se permite al estudiante un contacto con la realidad cercana, de tal forma que pueda diversificar sus aprendizajes pero también trasferir su conocimiento adquirido, le permite un alto nivel de dominio en cuestiones que tienen que ver con la sostenibilidad.

En cuanto a los resultados de la encuesta electrónica aplicada nuestros resultados de la tabla 2 y la figura 1 se desprende que la mayoría de los alumnos se muestran de acuerdo en relación con los ítems 1, 5, 7, 11, 12, 16 y 17. Estos ítems hacen referencia a competencias que pueden considerarse de un carácter más genérico y que se quedarían en el saber o saber cómo, por ejemplo:

-Ítem 1: Conozco la interrelación entre los sistemas naturales, sociales y económicos.

-Ítem 2: Considero la promoción del desarrollo humano sostenible como una finalidad fundamental de la formación ciudadana.

-Ítem 3: Sé valorar y analizar críticamente las consecuencias que mi actuación personal y profesional puede tener en el desarrollo integral de los educandos y en la promoción de un desarrollo humano sostenible.

En función del momento de aplicación, encontramos diferencias significativas en siete de las 18 competencias y resultados de aprendizaje.

Figura 1. Competencias y resultados de aprendizaje

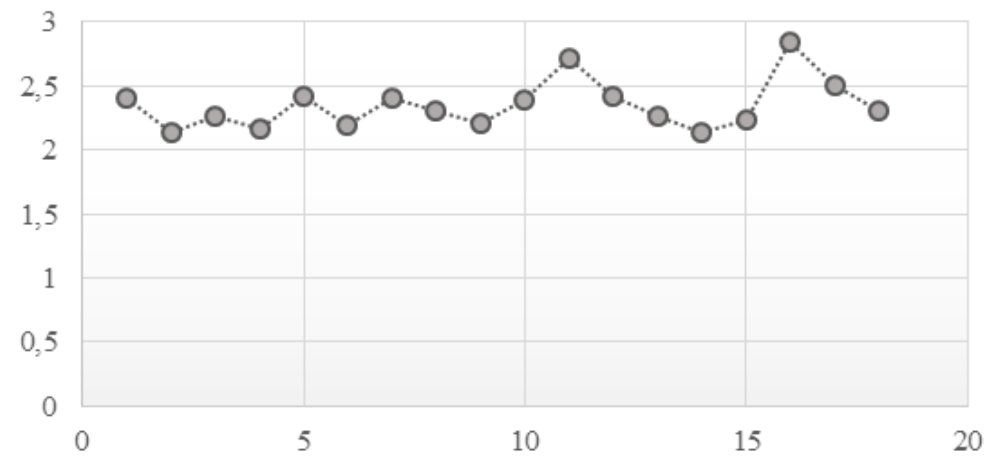


Tabla 2. Competencias y resultados de aprendizaje

\begin{tabular}{|c|c|c|c|c|c|c|c|c|}
\hline \multirow{2}{*}{\multicolumn{3}{|c|}{ COMPETENCIAS Y RESULTADOS DE APRENDIZAJE }} & \multirow{3}{*}{$\begin{array}{l}\text { Sx } \\
.805\end{array}$} & \multicolumn{4}{|c|}{$\%$} & \multirow{3}{*}{$\begin{array}{c}N \\
115\end{array}$} \\
\hline & & & & \multirow{2}{*}{$\frac{1}{11.3}$} & \multirow{2}{*}{$\frac{2}{45.2}$} & \multirow{2}{*}{$\frac{3}{34.8}$} & \multirow{2}{*}{$\frac{4}{8.7}$} & \\
\hline 1 & $\begin{array}{l}\text { Conozco la interrelación entre los sistemas naturales, } \\
\text { sociales y económicos }\end{array}$ & 2.41 & & & & & & \\
\hline 2 & $\begin{array}{l}\text { Comprendo y sé analizar las relaciones entre los sistemas } \\
\text { naturales y los sistemas sociales y económicos }\end{array}$ & 2.14 & .771 & 20.9 & 47.0 & 29.6 & 2.6 & 115 \\
\hline 3 & $\begin{array}{l}\text { Soy capaz de prever las repercusiones de los cambios en } \\
\text { los sistemas naturales, sociales y económicos }\end{array}$ & 2.27 & .753 & 15.7 & 44.3 & 37.4 & 2.6 & 115 \\
\hline 4 & $\begin{array}{l}\text { Conozco procedimientos y recursos para integrar la } \\
\text { sostenibilidad en las asignaturas }\end{array}$ & 2.16 & .733 & 17.4 & 52.2 & 27.8 & 2.6 & 115 \\
\hline 5 & $\begin{array}{l}\text { Sé analizar las oportunidades que se presentan en las } \\
\text { asignaturas para planificar proyectos educativos que } \\
\text { integren la sostenibilidad }\end{array}$ & 2.42 & .731 & 10.7 & 40.2 & 45.5 & 3.6 & 115 \\
\hline 6 & $\begin{array}{l}\text { Sé diseñar proyectos educativos desde la perspectiva de } \\
\text { la sostenibilidad }\end{array}$ & 2.19 & .782 & 18.3 & 48.7 & 28.7 & 4.3 & 115 \\
\hline 7 & $\begin{array}{l}\text { Sé identificar los posibles impactos socioambientales } \\
\text { derivados de mis actuaciones educativas }\end{array}$ & 2.41 & .805 & 13.0 & 40.0 & 40.0 & 7.0 & 115 \\
\hline 8 & $\begin{array}{l}\text { Sé cómo desarrollar actuaciones educativas que } \\
\text { minimicen impactos socioambientales negativos }\end{array}$ & 2.30 & .763 & 14.0 & 46.5 & 35.1 & 4.4 & 115 \\
\hline 9 & $\begin{array}{l}\text { Se diseñar y desarrollar actuaciones educativas teniendo } \\
\text { en cuenta los impactos socioambientales negativos e } \\
\text { incorporando acciones correctoras }\end{array}$ & 2.21 & .734 & 15.8 & 50.0 & 31.6 & 2.6 & 115 \\
\hline 10 & $\begin{array}{l}\text { Conozco programas educativos comunitarios que } \\
\text { fomentan la participación y el compromiso en la mejora } \\
\text { socioambiental }\end{array}$ & 2.39 & .886 & 18.3 & 33.0 & 40.0 & 8.7 & 115 \\
\hline 11 & $\begin{array}{l}\text { Sé desenvolverme de manera satisfactoria en proyectos } \\
\text { educativos comunitarios, fomentando la participación }\end{array}$ & 2.72 & .756 & 5.2 & 30.4 & 51.3 & 13.0 & 115 \\
\hline 12 & $\begin{array}{l}\text { Sé diseñar y llevar a cabo actividades socioeducativas en } \\
\text { procesos comunitarios participativos que promueven la } \\
\text { sostenibilidad, 1ntiéndome parte integrante de mi entorno }\end{array}$ & 2.42 & .848 & 13.9 & 40.0 & 36.5 & 9.6 & 115 \\
\hline 13 & Conozco los principios éticos de la sostenibilidad & 2.26 & .889 & 21.7 & 38.3 & 32.2 & 7.8 & 115 \\
\hline 14 & $\begin{array}{l}\text { Comprendo los principios éticos de la sostenibilidad y } \\
\text { soy capaz de integrarlos en mis acciones profelonales y } \\
\text { personales. }\end{array}$ & 2.14 & .857 & 24.3 & 43.5 & 26.1 & 6.1 & 115 \\
\hline 15 & $\begin{array}{l}\text { Se diseñar y/o gestionar proyectos educativos, tomando } \\
\text { en conlderación la ética ecológica, para mejorar la } \\
\text { calidad de vida y promover el bien común }\end{array}$ & 2.23 & .738 & 15.7 & 48.7 & 33.0 & 2.6 & 115 \\
\hline 16 & $\begin{array}{l}\text { Con1dero la promoción del desarrollo humano sostenible } \\
\text { como una finalidad fundamental de la formación } \\
\text { ciudadana }\end{array}$ & 2.84 & .904 & 7.8 & 26.1 & 40.0 & 26.1 & 115 \\
\hline 17 & $\begin{array}{l}\text { Sé valorar y analizar críticamente las consecuencias que } \\
\text { mi actuación personal y profelonal puede tener en el } \\
\text { desarrollo integral de los educandos y en la promoción de } \\
\text { un desarrollo humano sostenible }\end{array}$ & 2.51 & .754 & 7.8 & 40.9 & 43.5 & 7.8 & 115 \\
\hline 18 & $\begin{array}{l}\text { Sé diseñar y desarrollar propuestas de intervención } \\
\text { educativa que integren valores de sostenibilidad y } \\
\text { redunden en la justicia y el bien común }\end{array}$ & 2.30 & .761 & 12.2 & 52.2 & 29.6 & 6.1 & 115 \\
\hline
\end{tabular}

A partir del estudio de medias que se muestra en la tabla 3 se puede afirmar que los estudiantes de cuarto tienen mayor percepción que los estudiantes de primero en relación con la adquisición de competencias en sostenibilidad. 
Tabla 3. Diferencias por curso entre competencias y resultados de aprendizaje

\begin{tabular}{clccc}
\hline \multicolumn{1}{c}{ COMPETENCIAS Y RESULTADOS DE APRENDIZAJE } & Primero & Cuarto & Sig. \\
\hline 6 & Sé diseñar proyectos educativos desde la perspectiva de la sostenibilidad & 2.05 & 2.46 & .007 \\
\hline 8 & $\begin{array}{l}\text { Sé cómo desarrollar actuaciones educativas que minimicen impactos } \\
\text { socioambientales negativos }\end{array}$ & 2.20 & 2.50 & .046 \\
\hline 9 & $\begin{array}{l}\text { Se diseñar y desarrollar actuaciones educativas teniendo en cuenta los } \\
\text { impactos socioambientales negativos e incorporando acciones correctoras }\end{array}$ & 2.09 & 2.45 & .014 \\
\hline 11 & $\begin{array}{l}\text { Sé desenvolverme de manera satisfactoria en proyectos educativos } \\
\text { comunitarios, fomentando la participación }\end{array}$ & 2.61 & 2.95 & .020 \\
\hline 12 & $\begin{array}{l}\text { Sé diseñar y llevar a cabo actividades socioeducativas en procesos } \\
\text { comunitarios participativos que promueven la sostenibilidad, 1ntiéndome } \\
\text { parte integrante de mi entorno }\end{array}$ & 2.30 & 2.64 & .042 \\
\hline 16 & $\begin{array}{l}\text { Considero la promoción del desarrollo humano sostenible como una } \\
\text { finalidad fundamental de la formación ciudadana }\end{array}$ & 2.62 & 3.28 & .000 \\
\hline 18 & $\begin{array}{l}\text { Sé diseñar y desarrollar propuestas de intervención educativa que integren } \\
\text { valores de sostenibilidad y redunden en la justicia y el bien común }\end{array}$ & 2.16 & 2.56 & .006 \\
\hline
\end{tabular}

Por su parte, en función de la titulación se obtienen diferencias significativas en tres de las 18 competencias y resultados de aprendizaje. En el estudio de medias, podemos observar que los estudiantes de Educación Social afirman adquirir en mayor medida la competencia 11 y 16 respecto a los estudiantes de Pedagogía, mientras que estos afirman conocer más los principios éticos de la sostenibilidad (13) que los estudiantes de Ed. Social.

Tabla 4. Diferencias entre titulaciones respecto a competencias y resultados de aprendizaje

\begin{tabular}{cl}
\hline \multicolumn{1}{c}{ COMPETENCIAS Y RESULTADOS DE APRENDIZAJE } \\
\hline 11 & $\begin{array}{l}\text { Sé desenvolverme de manera satisfactoria en proyectos educativos } \\
\text { comunitarios, fomentando la participación }\end{array}$ \\
\hline 13 & Conozco los principios éticos de la sostenibilidad \\
\hline 16 & $\begin{array}{l}\text { Considero la promoción del desarrollo humano sostenible como una } \\
\text { finalidad fundamental de la formación ciudadana }\end{array}$ \\
\hline
\end{tabular}

Por último, a partir de los resultados mostrados en las tablas 5 y 6 , se puede afirmar que los estudiantes participantes en Proyectos de Sostenibilidad y que han cursado alguna materia específica al respecto, adquieren mejor las competencias en sostenibilidad que el resto. 
Tabla 5. Diferencias entre participantes y no en proyectos de Sostenibilidad respecto a competencias y resultados de aprendizaje

\begin{tabular}{clccc}
\hline & \multicolumn{1}{c}{$\begin{array}{c}\text { COMPETENCIAS Y RESULTADOS DE APRENDIZAJE } \\
\text { (Participación en Proyectos de Sostenibilidad) }\end{array}$} & No & Si & Sig. \\
\hline 1 & Conozco la interrelación entre los sistemas naturales, sociales y económicos & 2.34 & 2.92 & .014 \\
\hline 3 & $\begin{array}{l}\text { Soy capaz de prever las repercusiones de los cambios en los sistemas naturales, } \\
\text { sociales y económicos }\end{array}$ & 2.22 & 2.69 & .031 \\
\hline 7 & $\begin{array}{l}\text { Sé identificar los posibles impactos socioambientales derivados de mis } \\
\text { actuaciones educativas }\end{array}$ & 2.34 & 2.92 & .014 \\
\hline 12 & $\begin{array}{l}\text { Sé diseñar y llevar a cabo actividades socioeducativas en procesos comunitarios } \\
\text { participativos que promueven la sostenibilidad, 1ntiéndome parte integrante de mi } \\
\text { entorno }\end{array}$ & 2.33 & 3.08 & .003 \\
\hline 14 & $\begin{array}{l}\text { Comprendo los principios éticos de la sostenibilidad y soy capaz de integrarlos en } \\
\text { mis acciones profelonales y personales. }\end{array}$ & 2.04 & 2.92 & .000 \\
\hline 15 & $\begin{array}{l}\text { Se diseñar y/o gestionar proyectos educativos, tomando en con1deración la ética } \\
\text { ecológica, para mejorar la calidad de vida y promover el bien común }\end{array}$ & 2.15 & 2.85 & .001 \\
\hline 16 & $\begin{array}{l}\text { Considero la promoción del desarrollo humano sostenible como una finalidad } \\
\text { fundamental de la formación ciudadana }\end{array}$ & 2.73 & 3.77 & .000 \\
\hline
\end{tabular}

Tabla 6. Diferencias estudiantes que han recibido Formación Docente Previa en Sostenibilidad respecto a competencias y resultados de aprendizaje

\begin{tabular}{clccc}
\hline \multicolumn{1}{c}{$\begin{array}{c}\text { COMPETENCIAS Y RESULTADOS DE APRENDIZAJE } \\
\text { (Formación Docente Previa en Sostenibilidad) }\end{array}$} & No & Si & Sig. \\
\hline 1 & Conozco la interrelación entre los sistemas naturales, sociales y económicos & 2.37 & 2.69 & .014 \\
\hline 3 & $\begin{array}{l}\text { Soy capaz de prever las repercusiones de los cambios en los sistemas } \\
\text { naturales, sociales y económicos }\end{array}$ & 2.25 & 2.46 & .031 \\
\hline 7 & $\begin{array}{l}\text { Sé identificar los posibles impactos socioambientales derivados de mis } \\
\text { actuaciones educativas }\end{array}$ & 2.38 & 2.62 & .014 \\
\hline 8 & $\begin{array}{l}\text { Sé cómo desarrollar actuaciones educativas que minimicen impactos } \\
\text { socioambientales negativos }\end{array}$ & 2.27 & 2.54 & .048 \\
\hline 12 & $\begin{array}{l}\text { Sé diseñar y llevar a cabo actividades socioeducativas en procesos } \\
\text { comunitarios participativos que promueven la sostenibilidad, 1ntiéndome } \\
\text { parte integrante de mi entorno }\end{array}$ & 2.39 & 2.62 & .003 \\
\hline 14 & $\begin{array}{l}\text { Comprendo los principios éticos de la sostenibilidad y soy capaz de } \\
\text { integrarlos en mis acciones profelonales y personales. }\end{array}$ & 2.14 & 2.15 & .000 \\
\hline 15 & $\begin{array}{l}\text { Se diseñar y/o gestionar proyectos educativos, tomando en conlderación la } \\
\text { ética ecológica, para mejorar la calidad de vida y promover el bien común }\end{array}$ & 2.21 & 2.38 & .001 \\
\hline 16 & $\begin{array}{l}\text { Considero la promoción del desarrollo humano sostenible como una finalidad } \\
\text { fundamental de la formación ciudadana }\end{array}$ & 2.79 & 2.23 & .000 \\
\hline 18 & $\begin{array}{l}\text { Sé diseñar y desarrollar propuestas de intervención educativa que integren } \\
\text { valores de sostenibilidad y redunden en la justicia y el bien común }\end{array}$ & 2.25 & 2.62 & .005 \\
\hline
\end{tabular}

\section{DISCUSIÓN Y CONCLUSIONES}

Partiendo de la premisa de que el futuro será sostenible o no será, la Universidad como encargada de formar a los profesionales que tomarán las riendas de nuestra sociedad en un futuro, debe adquirir un fuerte compromiso con la enseñanza de competencias en sostenibilidad de forma transversal. Desde la perspectiva que venimos defendiendo, ser hoy un buen ciudadano significa, necesariamente, ser un buen 
ciudadano del planeta Tierra. La Educación para el Desarrollo Sostenible tiene entre sus funciones contribuir a esta meta, lo cual le exige replantear una de las finalidades que han sido atribuidas a la educación por las sociedades democráticas en el último siglo: la formación de ciudadanos democráticos y ambientalmente sostenibles (Murga-Menoyo y Novo, 2017).

El proyecto EDINSOST investiga sobre qué estrategias serían las adecuadas para poder introducir la sostenibilidad dentro del sistema universitario español, a través de los estudios realizados y que hemos mostrado a lo largo del documento, podemos extraer que el mapa de competencias presentado está diseñado de tal forma que nos permite integrar conocimientos, actitudes y valores, en harmonía con un futuro sostenible y es de gran utilidad para analizar las prácticas docentes en el aula (Sánchez et al., 2018).

Como muestran los resultados del cuestionario, se hace necesaria la formación en sostenibilidad. Los alumnos afirman que no tienen formación suficiente en materia de sostenibilidad y se comprueba como los alumnos que participan en proyectos y tienen docencia específica en materias de sostenibilidad adquieren mejor las competencias en sostenibilidad que el resto, lo cual viene a corroborar la necesidad expresada por Michelsen (2016) de formar en materia de sostenibilidad a los ciudadanos y profesionales del futuro. Además, tal y como se expresa en el mapa de competencias, se precisa de la utilización de metodologías docentes que vayan más allá del saber, y pasen a utilizar estrategias que permitan a los alumnos llegar a un saber cómo y saber hacer. Es de vital importancia que la formación en sostenibilidad se dirija no solo a la adquisición de unos conocimientos, sino a que los estudiantes sean capaces de aplicar los criterios de Sostenibilidad en cualquiera de sus competencias y tareas profesionales independientemente de su área de conocimiento. Esto nos lleva directamente al repensar del curriculum académico (Murga-Menoyo y Novo, 2015), pues este debe ser abordado, ya no solo desde metodologías que contemplen los tres niveles de dominio, sino también desde una perspectiva interdisciplinar (Aznar y Barrón, 2017).

Es precisamente en esta segunda parte donde debe caer la mayor parte de la responsabilidad. "El problema es si el profesorado está preparado para eso porque, claro, cómo nos van a formar si a lo mejor ellos no saben lo que es la sostenibilidad", (Valderrama-Hernández et al., 2019). El alumnado no percibe que sus profesores estén preparados para entender la sostenibilidad, por lo tanto, mucho menos para integrarla en las clases de manera transversal o de manera específica. La visión de las alumnas y alumnos en diversas Universidades de España es común en cuanto al profesorado.

No se puede obviar que la responsabilidad universitaria en desarrollo sostenible está actualmente en cambio de paradigma liderado por los alumnos de diferentes universidades europeas. Por lo tanto, es en este sector universitario donde reside el liderazgo y por lo tanto el cambio y avance del desarrollo sostenible en la 
Universidad. Segalas y Sánchez (2019) muestran cómo los alumnos consideran necesario que en la universidad se realice formación en sostenibilidad, y consideran que su propia formación es escasa y que en su titulación no se trabajan apropiadamente las competencias en sostenibilidad. Creen que la motivación del profesorado es un aspecto fundamental para que la sostenibilidad se trabaje en una titulación.

Ante este escenario una línea de futuro sobre la que se debe profundizar es que la limitación por parte de la universidad podría localizarse en mayor medida en el profesorado. Con metodologías que no contemplan la sostenibilidad en la docencia ni en la práctica universitaria diaria. Los alumnos no perciben a los profesores (solo a los que imparten materias relacionadas con educación ambiental) como formadores en sostenibilidad. No hay una preocupación ni una diligencia en cambiar la docencia, y por lo tanto la universidad hacia un modelo sostenible y garantista.

\section{REFERENCIAS}

Andrades, F.J., Larrán, M., y Muriel, M.J. (2018). Analysing the incorporation of sustainability themes into the university curricula: a case study of a Spanish public university. International Journal of Sustainable Development \& World Ecology, 3(1), 642-654.

Aznar, P., y Barrón, A. (2017). El desarrollo humano sostenible: un compromiso educativo. Teoría de la Educación, 29(1), 22-53.

Boni, A., Hofmann, A., y Sow, J (2012). Educando para una ciudadanía global. Una experiencia de investigación cooperativa entre docentes y profesionales de las ONGD. Estudios sobre Educación, 23, 63-81.

Campbell, D.T., y Stanley, J.C. (1963). Experimental and quasi-experimental designs for research. Chicago: Rand McNally \& Company.

Caride, J.A. (2017). Educación social, derechos humanos y sostenibilidad en el desarrollo comunitario. Teoría de la Educación, 29(1), 245-272.

Sánchez, F., Segalás, J., Vidal, E., Martín, C., Climent, J., López, D., y Cabré, J.M. (2018). Improving engineering educators' sustainability competencies by using competency maps: The EDINSOST project. International Journal of Engineering Education, 34(5), $1527-1537$.

CONGCYL (2014). Posicionamiento sobre la importancia de la Educación para el Desarrollo y el rol de las ONGD en la construcción de una ciudadanía global. Valladolid: CONGCYL.

Larrán, M., Herrera, J., y Andrades, F. (2015). Factors influencing the presence of sustainability initiatives in the strategic planning of Spanish universities. Environmental Education Research, 21(8), 1155-1187.

Martín, J., y Caballero, D. (2017). La Cooperación Oficial europea y española ante el nuevo escenario del sistema internacional de cooperación al desarrollo. Acciones $e$ Investigaciones Sociales, 37, 7-25.

Michelsen, G. (2016). Policy, politics and polity in higher education for sustainable development. En M. Barth, G. Michelsen, T. Rieckmann e I. Routledge (Eds), Handbook of Higher Education for Sustainable Development (pp. 40-55). New York: Routledge.

Murga-Menoyo, M.A. y Novo, M. (2015). The processes of integrating sustainability in higher education curricula: A theoretical-practical experience regarding key competences and 
their cross-curricular incorporation into degree courses. En W. Leal Filho (Ed.), Transformative Approaches to Sustainable Development at Universities (pp. 119-135). Cham: Springer International Publishing.

Murga-Menoyo, M.A. y Novo, M. (2017). Sostenibilidad, desarrollo «glocal» y ciudadanía planetaria. Revista Teoría de la Educación, 29, 55-78.

Naciones Unidas (2012). The future we want: Outcome document of the United Nations Conference on Sustainable Development adopted. Rio de Janeiro: United Nations.

Naciones Unidas (2014) El camino hacia la dignidad para 2030: acabar con la pobreza y transformar vidas protegiendo el planeta. Nueva York: United Nations.

Núñez, B., Calvo, R., y Álvarez, J.M. (2015). Los proyectos de cooperación: una actividad de tiempo libre de alumnos universitarios. Pedagogía Social: Revista Interuniversitaria, 25, 201-220. doi:10.7179/PSRI_2015.25.9

Ortega, M.L. (2007). Estrategia de Educación para el Desarrollo de la Cooperación Española. Madrid: Ministerio de Asuntos Exteriores y de Cooperación.

Sainz, J. (2016). El papel de las Enseñanzas Técnicas Universitarias en la Cooperación Universitaria al Desarrollo y en la formación de ciudadanía global y transformadora. Revista de Didáctica: IKASTORRATZA, 17, 1-7.

Sánchez, F., Segalas, J., Cabré, J., Climent, J., López, D., Martín, C., y Vidal, E. (2017). El proyecto EDINSOST: Inclusión de los ODS en la educación superior. Revista Española de Desarrollo y Cooperación, 41, 67-81.

Segalas, J., y Sánchez, F. (2019). El proyecto EDINSOST. Formación en las Universidades españolas de profesionales como agentes de cambio para afrontar los retos de la sociedad. Revista de Educación Ambiental y Sostenibilidad, 1(1), 1204.

Valderrama-Hernández, R., Alcántara, L., Sánchez-Carracedo, F., Caballero, D., Gil-Doménech, D., Serrate, S., Vidal-Raméntol, S., y Miñano, R. (2019). ¿Forma en sostenibilidad el sistema universitario español? Visión del alumnado de cuatro universidades. Educación $X X 1,22(1), 1-26$.

Recibido: 20 de agosto de 2019

Recepción Modificaciones: 3 de septiembre de 2019

Aceptado: 17 de septiembre de 2019 